\title{
Role of Vitamin D in COVID-19
}

\author{
Amit Samadhiya ${ }^{1}$, Rajeev Goyal ${ }^{2}$, Aditi Singh ${ }^{3}$, Santosh K Gupta ${ }^{4}$
}

\begin{abstract}
Aim: To review the role of vitamin D in COVID-19.

Background: The COVID-19 pandemic has caused a tremendous social and economic impact worldwide, and rapid outspreading of severe acute respiratory syndrome coronavirus 2 (SARS-CoV-2) is still being seen in India. Currently, there are two explanations available for the fatal ARDS in COVID-19 cases. Cytokine storm is characterized by unregulated release of various proinflammatory cytokines, i.e., TNF- $a$, INF- $\gamma$, IL2, IL6, IL8, IL12, CCL2, CCL3, and CXCL10, after interaction between SARS-CoV-2 and host immune cells. On the other hand, bradykinin storm is happening due to downregulation of ACE in the lung tissue. Immunomodulatory effects of vitamin D is proved through various studies. There is no individual therapy and vaccination for the SARS-Cov-2; thus, repurposing of available medicines is crucial now. Antiviral, anti-inflammatory, and immuno-boosting effects of vitamin D has proven in many studies. These effects are very relevant for its putative beneficial effect in SARS-CoV-2. Review result: Vitamin D has already been used as a repurposed drug in $\mathrm{H} 5 \mathrm{~N} 1$ pneumonia. Immunomodulatory and antibacterial role of vitamin $D$ is well established. There are studies suggesting toward the cytokine storm as a main culprit behind deadly ARDS in COVID-19. Vitamin D was found as an anti-inflammatory and lung protective substance. Vitamin D was also found protective against bacterial LPS-induced injury by increasing expression of ACE-II and producing antibacterial protein cathelicidin.

Conclusion: Before COVID-19 pandemic, we realized the silent pandemic of vitamin D deficiency. The immunomodulatory, anti-inflammatory, and antibacterial role of vitamin D has been shown in many studies. There are studies which have demonstrated the inverse relationship between vitamin D level and susceptibility to COVID-19. Vitamin-D also plays a crucial role in limiting the fibrosis in the damaged pulmonary tissue and also responsible for deciding overall morbidity of the patient. Thus, it is imperative to think about the potential of vitamin $\mathrm{D}$ as a repurposed drug for COVID-19 cases.
\end{abstract}

Keywords: ARDS, ACE-II, COVID-19, Cytokine storm, SARSCov-2, Vitamin D.

Indian Journal of Medical Biochemistry (2020): 10.5005/jp-journals-10054-0150

\section{BACKGROUND}

COVID-19 is rapidly spreading in India now, resulting in more than 90,000 cases per day and taking a toll of more than 1,000 lives per day. ${ }^{1}$ COVID-19 is primarily a respiratory disease transmitted through person to person by air droplets and infected fomites. Major presenting symptoms are sore throat, fever, anosmia, breathing difficulty, myalgia, diarrhea, and confusion. Common risk factors associated with the COVID-19 are old age, diabetes, obesity, and hypertension. ${ }^{2,3}$

SARS-CoV-2 enters in type-2 pneumocytes through ACE-2 receptors with the help of its spike protein " $S$ ". This complex is endocytosed, and positive-sense SSRNA is released inside the cell. ACE-2 receptors are present on type 2 pneumocytes, enterocytes of all parts of the small intestine, the brush border of the proximal tubular cells of the kidney, as well as the endothelial cells of small and large arteries and veins and on the arterial smooth muscle cells. Tissue tropism of SARS-CoV-2 indicates that the disease was more likely a systematic disease rather than a local pulmonary disease.

Most of the infected patients clear viral load by second week of illness and seroconversion takes place in almost $100 \%$ patients with in 2-4 weeks. ${ }^{4}$

\section{ARDS IN COVID-19}

SARS-CoV-2 usually cause mild to moderate disease in most cases but in many, with risk factors, it develops into a life-threatening ARDS. This severe respiratory illness is a hallmark of coronavirusassociated diseases previously caused by SARS and MERS. ${ }^{5}$

Various explanations came in light to explain the pathophysiology of acute respiratory distress syndrome in COVID-19

\footnotetext{
${ }^{1-3}$ Department of Biochemistry, Lady Hardinge Medical College, Delhi, India

${ }^{4}$ Department of Biochemistry, Maulana Azad Medical College, Delhi, India

Corresponding Author: Santosh K Gupta, Department of Biochemistry, Maulana Azad Medical College, Delhi, India, Phone: +91 9968604224, e-mail: skgupta1952@rediffmail.com

How to cite this article: Samadhiya A, Goyal R, Singh A, et al. Role of Vitamin-D in COVID-19. Indian J Med Biochem 2020;24(3):130-133.

Source of support: Nil

Conflict of interest: None
}

patients. Earlier research suggested about the cytokine storm created by innate immune response after interaction between pattern recognition receptors (PRRs) of immune cells and pathogenassociated molecular patterns (PAMPs) associated with SARS-CoV-2. Cytokine storm is characterized by disproportionate release of both T helper1 (Th1) and T helper2 (Th2) cytokines including IL-1, IL-1B, IL-2, IL-6, IL-7, IL-8, IL-10, IL-12, IL-13, IL-17, granulocyte colonystimulating factor (GCSF), interferon- $\gamma$ inducible protein 10 (IP-10), macrophage inflammatory protein 1-a (MIP-1a), tumor necrosis factor- $a$ (TNF- $a)$, monocyte chemoattractant protein 1 (MCP-1), and IFN- $\gamma$ through activation of various transcription factors IRF3, IRF7, STAT, and NF-KB. 6,7

Very recently, supercomputer analysis of gene expression data from cells in bronchoalveolar lavage fluid (BALF) from COVID19 patients when compared to BALF from controls identified a 
critical imbalance in RAS represented by decreased expression of angiotensin converting enzyme (ACE) in combination with increases in ACE-2, renin, angiotensin, key RAS receptors, kininogen, and many kallikrein enzymes that activate it, and both bradykinin receptors. ${ }^{8}$

Bradykinin is a nine amino acid peptide that is degraded by ACE, while ACE-2 promotes its synthesis through angiotensin-II (AT-II) degradation products. Decreased expression of ACE in COVID-19 patients may cause excess of bradykinin referred to as a bradykinin storm. This bradykinin storm purportedly increases vascular permeability and causes neutrophilic infiltration by CXCR5 chemokine. Together with other mechanisms of cell injury and cytokine release, this bradykinin storm culminates in ARDS. Bradykinin storm explains the temporal association between presence of dry cough, fatigue, headache, muscle pain, and diarrhea as a pathognomonic symptoms and natural history of illness in a more rational way.

\section{Role of Vitamin D in COVID-19}

There is no specific therapy and vaccine available at present for the SARS-CoV-2. Thus, rethinking the possible role of available medicines is crucial now, both for the prevention and reducing the severity of the COVID-19. Vitamin D is one such nutrient with medicinal values. Many research studies have shown the antiviral, anti-inflammatory, and immune-boosting effects of vitamin $D$, which can interfere directly with viral replication. It also acts as an immunomodulatory agent to curb the outburst of cytokines. ${ }^{9-11}$

Vitamin D decreases the production of Th1 cells. Thus, it can suppress the progression of inflammation through suppression of inflammatory cytokines such as IL-6, IL-8, IL-12, and IL-17. ${ }^{12,13}$ Vitamin $D$ also decreases the generation of TNFa and production of nuclear factor-KB (NFKB) which is a key transcription factor behind the synthesis of proinflammatory cytoines. ${ }^{14}$

There are many studies which suggest that vitamin D increases the expression of ACE-2 that acts as an entry point for SARS-CoV-2. Calcitriol (1,25-dihydroxyvitamin D3) enhances the expression of ACE-2 by its impact on ACE2/Ang(1-7)/MasR pathway. ${ }^{15}$ Another study found that activated vitamin $D$ exhibits reno-protective effect by decreasing ACE-1 and the ratio of ACE-1/ACE2 in streptozotocininduced diabetic nephropathic rats. Vitamin $D$ treatment also activated ACE-2 expression in kidney tubule cells. ${ }^{16}$

These findings of increased ACE-2 expression by calcitriol create a dilemma about the beneficial role of vitamin D in COVID19 patients.

A study reported that vitamin $D$ receptor-lacking mice had elevated levels of renin and angiotensin II (Ang II). Renin is a proteolytic enzyme and a positive regulator of Ang II. Vitamin $D$ is a potent inhibitor of renin. ${ }^{17}$ Vitamin $D$ supplement was shown to prevent the accumulation of Ang II and to decrease the proinflammatory activity of Ang II by suppressing the release of renin in patients infected with COVID, thus reducing the risk of ARDS, myocarditis, or cardiac injury. ${ }^{18}$

Pulmonary fibrosis is frequently seen in many severe cases of COVID-19 following recovery of acute disease. Ang II was found in high levels in bleomycin-treated mice and in patients with pulmonary fibrosis TGF- $\beta_{1}$ is activated by angiotensin-II and serves as a signal to activate the intrinsic apoptosis pathway in bleomycin-induced pulmonary fibrosis. Thus, accumulation of Ang II may be a responsible factor for both apoptosis and fibrosis..$^{19,20}$ Ang II is degraded by ACE-2, which cleaves its carboxy terminal amino acid to form a seven amino acid peptide ANG1-7. ANG1-7 acts by its receptor mas to suppress bleomycin-induced fibrosis by downregulating the activation of JNK. JNK is required for bleomycin and angiotensin II-induced apoptosis. ${ }^{21,22}$ Calcitriol could prove effective in reducing the risk of fibrosis by interfering with Ang II accumulation. It may also decrease the extent and severity of fibrosis after healing of the severely inflamed lungs.

Interestingly, many of the risk factors for vitamin D deficiency and COVID-19 infection are common, i.e., old age, obesity, dark skin pigmentation, chronic kidney disease (CKD), and winter season. Recent reports have indicated that those residing at higher latitudes or with darker skin pigmentation (Black Asian Minority ethnics - BAME in UK) may be particularly affected by COVID-19. ${ }^{23}$ It is already evident that there is a worldwide association between northern latitude and increased COVID-19 mortality. ${ }^{24}$ Thus, vitamin D status could be impacting the COVID-19 severity. Vitamin D's role has been reported in many conditions associated with pneumonia, cytokine hyperproduction, and ARDS. ${ }^{25}$

Univariate analysis conducted in a health insurance service company's employee demonstrated an association between low plasma 25(OH)D level and increased likelihood of COVID-19 infection. $^{26}$

However, another study conducted by UK BioBank did not show any relation between vitamin $D$ concentrations and risk of COVID-19 infection nor the vitamin D concentration explained the ethnic differences in COVID-19 infection. ${ }^{27}$

Vitamin D has recently been used as a repurposed drug for influenza A H5N1 virus-induced pulmonary disease. ${ }^{28}$ Studies demonstrated the benefit of vitamin $\mathrm{D}$ as an adjuvant therapy along with antiretroviral agents in HIV-infected patients. ${ }^{29,30}$ Calcitriol pretreatment was effective in animal models of ARDS, decreasing lung permeability by modifying of renin-angiotensin system activity and ACE 2 expression. ${ }^{31}$ Vitamin D deficiency was found to be associated with increased morbidity. A study was conducted retrospectively on 134 patients with COVID-19. In this study, prevalence of vitamin D deficiency, compliance with local treatment protocol, and relationship of baseline serum $25(\mathrm{OH}) \mathrm{D}$ with markers of COVID-19 severity were analyzed. It was found that prevalence of vitamin D deficiency was higher in patients requiring admission in a critical care unit in comparison to patients managed in medical wards, but there was no association with fatality. ${ }^{32}$

Vitamin D has immunomodulatory properties, which include downregulation of proinflammatory cytokines, attenuation of lipopolysaccharide-induced acute lung injury in mice by blocking effects on the angiopoietin signaling pathway, and on the reninangiotensin pathway. ${ }^{33}$ Both in a mouse model of bleomycininduced interstitial pneumonia and in human cell lines, vitamin D3 is locally activated in lung tissue and has a preventive effect on experimental interstitial pneumonitis. ${ }^{34}$ Interestingly, vitamin $D$ does not cause overexpression of ACE-2 when given alone but it significantly reduced LPS-mediated underexpression of ACE-2. ACE-2 is in itself protective for pulmonary tissue because it degrades angiotensin-II which is a vasoactive and pro-inflammatory substance.

Vitamin $D$ is well known to modulate host immune responses through the production of the antimicrobial peptides, such as cathelicidin and defensins. ${ }^{35}$ Cathelicidin and defensin reduce the risk of secondary bacterial infections. It is seen that lipopolysaccharides (LPS) produced by secondary bacterial infections may potentially promote the cytokine storm. Thus, calcitriol could prove to be protective against LPS-mediated augmentation of cytokine IL6, IL12. ${ }^{36}$ 
Vitamin D in many ways modulates the host immune response and provides protection against deadly cytokine storm and reduces risk for ARDS. ${ }^{29}$ There is also evidence from a meta-analysis that regular oral vitamin $D$ intake is protective against respiratory tract infection, especially in vitamin D-deficient subjects. ${ }^{37,38} \mathrm{~A}$ significant negative correlation between mean levels of vitamin $D$ in each northern European countries and the number of COVID-19 cases per million population were observed. Vitamin D levels were severely low in the aging population, especially in Spain, Italy, and Switzerland. ${ }^{39,40}$ Mok et al. demonstrated in an in vitro experiment that the active form of vitamin D, calcitriol, exhibits significant potent activity against SARS-CoV-2. ${ }^{41}$ Hence, there is a potential role of vitamin D supplementation in COVID-19 patients.

\section{Conclusion}

It therefore seems plausible that serum vitamin D level may contribute to reducing the severity of illness caused by SARSCoV-2, particularly in settings where hypovitaminosis $D$ is frequent. Considering the widespread high prevalence of vitamin D deficiency in our country and proven immunomodulatory, anti-inflammatory, and anti-bacterial properties of activated vitamin $D$, this seems very pragmatic to evaluate the role of vitamin $D$ as a preventive measure and adjuvant treatment method through a large study. It would be prudent to start supplementing vitamin D to the high-risk individuals and fortifying the food products, following the "primum non nocere" principle of Hippocrates.

\section{References}

1. WHO, Coronavirus disease (COVID-2019) situation reports. 2020. https://www.who.int/emergencies/diseases/novel-coronavirus-2019/ situation-reports Accessed 18 Sept 2020.

2. Li H, Wang Y, Ji M, et al. Transmission routes analysis of SARS-CoV-2: a systematic review and case report. Front Cell Dev Biol 2020;8:618. DOI: $10.3389 /$ fcell.2020.00618.

3. Chen N, Zhou M, Dong X, et al. Epidemiological and clinical characteristics of 99 cases of 2019 novel coronavirus pneumonia in Wuhan, China: a descriptive study. Lancet 2020;395(10223):507-513. DOI: 10.1016/S0140-6736(20)30211-7.

4. Long Q-X, Liu B-Z. Antibody responses to SARS-CoV-2 in patients with COVID-19. Nat Med 2020;26(6):845-848. DOI: 10.1038/s41591020-0897-1.

5. Lau YL, Malik Peiris JS. Pathogenesis of severe acute respiratory syndrome. Curr Opin Immunol 2005;17(4):404-410. DOI: 10.1016/j. coi.2005.05.009.

6. Conti P, Ronconi G, Caraffa A, et al. Induction of pro-inflammatory cytokines (IL-1 and IL-6) and lung inflammation by coronavirus-19 (COVI-19 or SARS-CoV-2): anti-inflammatory strategies. J Biol Regul Homeost Agents 2020;34(2):327-331. DOI: 10.23812/CONTI-E.

7. Chen C, Zhang XR, Ju ZY, et al. Advances in the research of cytokine storm mechanism induced by corona virus disease 2019 and the corresponding immunotherapies. Zhonghua Shaoshang Zazhi 2020;36(6):471-475.

8. Garvin MR, Alvarez C, Miller Jl, et al. A mechanistic model and therapeutic interventions for COVID-19 involving a RASmediated bradykinin storm. eLife 2020;9:e59177. DOI: 10.7554/eLife. 59177.

9. Zhang Y, Leung DYM, Richers BN, et al. Vitamin D inhibits monocyte/ macrophage proinflammatory cytokine production by targeting MAPK phosphatase-1. J Immunol 2012;188(5):2127-2135. DOI: 10.4049/jimmunol.1102412.

10. Greiller $\mathrm{CL}$, Martineau AR. Modulation of the immune response to respiratory viruses by vitamin D. Nutrients 2015;7(6):4240-4270. DOI: 10.3390/nu7064240.
11. Zdrenghea MT, Makrinioti $\mathrm{H}$, Bagacean $\mathrm{C}$, et al. Vitamin $\mathrm{D}$ modulation of innate immune responses to respiratory viral infections. Rev Med Virol 2017;27(1). DOI: 10.1002/rmv.1909.

12. Ardizzone $S$, Cassinotti $A$, Trabattoni $D$, et al. Immunomodulatory effects of 1,25-dihydroxyvitamin D3 on TH1/TH2 cytokines in inflammatory bowel disease: an in vitro study. Int J Immunopathol Pharmacol 2009;22(1):63-71. DOI: 10.1177/039463200902200108.

13. Palmer MT, Lee $Y K$, Maynard CL, et al. Lineage-specific effects of 1,25-dihydroxyvitamin $D(3)$ on the development of effector CD4 T cells. J Biol Chem 2011;286(2):997-1004. DOI: 10.1074/jbc. M110.163790.

14. Peterson CA, Heffernan ME. Serum tumor necrosis factor-alpha concentrations are negatively correlated with serum $25(\mathrm{OH}) \mathrm{D}$ concentrations in healthy women. J Inflamm (Lond) 2008;5(1):10. DOI: 10.1186/1476-9255-5-10.

15. Cui C, Xu P, Li G, et al. Vitamin D receptor activation regulates microglia polarization and oxidative stress in spontaneously hypertensive rats and angiotensin II-exposed microglial cells: role of renin-angiotensin system. Redox Biol 2019;26:101295. DOI: 10.1016/j.redox.2019.101295.

16. Lin $M, G a o ~ P, Z$ Zhao $T$, et al. Calcitriol regulates angiotensin-converting enzyme and angiotensin converting-enzyme 2 in diabetic kidney disease. Mol Biol Rep 2016;43(5):397-406. DOI: 10.1007/s11033-0163971-5.

17. Li YC, Qiao G, Uskokovic M, et al. Vitamin D: a negative endocrine regulator of the renin-angiotensin system and blood pressure. J Steroid Biochem Mol Biol 2004;89-90(1-5):387-392. DOI: 10.1016/j. jsbmb.2004.03.004.

18. Hanff TC, Harhay MO, Brown TS, et al. Is there an association between COVID-19 mortality and the renin-angio-tensin system-a call for epidemiologic investigations. Clin Infect Dis 2020(15):ciaa329. DOI: 10.1093/cid/ciaa329.

19. Li X, Molina-Molina M, Abdul-Hafez A, et al. Angiotensin converting enzyme-2 is protective but downregulated in human and experimental lung fibrosis. Am J Physiol Lung Cell Mol Physiol 2008;295(1):L178-L185. DOI: 10.1152/ajplung.00009.2008.

20. LiX, Zhang H, Soledad-Conrad V, et al. Bleomycin-induced apoptosis of alveolar epithelial cells requires angiotensin synthesis de novo. Am J Physiol Lung Cell Mol Physiol 2003;284(3):L501-L507. DOI: 10.1152/ ajplung.00273.2002.

21. Bader M. Tissue renin-angiotensin-aldosterone systems: targets for pharmacological therapy. Annu Rev Pharmacol Toxicol 2010;50(1):439-465. DOI: 10.1146/annurev.pharmtox.010909.105610.

22. Lee VY, Schroedl C, Brunelle JK, et al. Bleomycin induces alveolar epithelial cell death through JNK-dependent activation of the mitochondrial death pathway. Am J Physiol Lung Cell Mol Physiol 2005;289(4):L521-L528. DOI: 10.1152/ajplung.00340.2004.

23. Laird E, Rhodes J, Kenny RA. Vitamin D and inflammation: potential implications for severity of COVID-19. Ir Med J 2020;113:81.

24. Mark. B. Latitude dependence of the COVID-19 mortality rate-A possible relationship to vitamin D deficiency? SSRN Electro J 2020;26:3561958.

25. Teymoori-Rad M, Shokri F, Salimi V, et al. The interplay between vitamin D and viral infections. Rev Med Virol 2019;29(2):e2032. DOI: 10.1002/rmv.2032.

26. Merzon E, Tworowski D, Gorohovski A, et al. Low plasma 25(OH) vitamin D level is associated with increased risk of COVID-19 infection: an Israeli population-based study. MedRxiv 2020. DOI: 10.1101/2020.07.01.20144329.

27. Hastie CE, Mackay DF, Ho F, et al. Vitamin D concentrations and COVID19 infection in UK Biobank. Diabetes MetabSyndr 2020;14(4):561-565. DOI: $10.1016 / j . d s x .2020 .04 .050$.

28. Huang $F$, Zhang $C$, Liu Q, et al. Identification of amitriptyline $\mathrm{HCl}$, flavin adenine dinucleotide, azacitidine and calcitriol as repurposing drugs for influenza A H5N1 virus-induced lung injury. PLoS Pathog 2020;16(3):e1008341. DOI: 10.1371/journal.ppat.1008341.

29. Jiménez-Sousa MÁ, Martínez I, Medrano LM, et al. Vitamin D in human immunodeficiency virus infection: influence on immunity and disease. Front Immunol 2018;9:458. DOI: 10.3389/fimmu.2018.00458. 
30. Jiménez-Sousa MA, Jiménez JL, Fernández-Rodríguez A, et al. VDR rs2228570 polymorphism is related to non-progression to AIDS in antiretroviral therapy naïve HIV infected patients. J Clin Med 2019;8(3):E311. DOI: 10.3390/jcm8030311.

31. Kong J, Zhu X, Shi Y, et al. VDR attenuates acute lung injury by blocking Ang-2 pathway and rennin angiotensin system. Mol Endocrino 2013;27(12):2116-2125. DOI: 10.1210/me.2013-1146.

32. Panagiotou $G$, Tee $S A$, Ihsan $Y$, et al. Low serum 25 -hydroxyvitamin $D$ (25[OH]D) levels in patients hospitalised with COVID-19 are associated with greater disease severity. Clin Endocrinol (Oxf) 2020(4). DOI: 10.1111/cen.14276.

33. Xu J, Yang J, Chen J, et al. Vitamin D alleviates lipopolysaccharideinduced acute lung injury via regulation of the renin angiotensin system. Mol Med Rep 2017;16(5):7432-7438. DOI: 10.3892/ mmr.2017.7546.

34. Tsujino I, Ushikoshi-Nakayama R, Yamazaki T, et al. Pulmonary activation of vitamin D3 and preventive effect against interstitial pneumonia. J Clin BiochemNutr 2019;65:245-251.

35. Croy HE, Abrahams SJ, Raed A, et al. Cathelicidin antimicrobial protein, vitamin $D$, and risk of death in critically ill patients. Crit Care 2015;19(1):80. DOI: 10.1186/s13054-015-0812-1.
36. Xiong $Y$, Liu $Y$, Cao L, et al. Transcriptomic characteristics of bronchoalveolar lavage fluid and peripheral blood mononuclear cells in COVID-19 patients. Emerg Microbes Infect 2020;9(1):761-770. DOI: $10.1080 / 22221751.2020 .1747363$.

37. Martineau AR, Jolliffe DA, Hooper RL, et al.Vitamin D supplementation to prevent acute respiratory tract infections: systematic review and meta-analysis of individual participant data. BMJ 2017;356:i6583. DOI: 10.1136/bmj.i6583.

38. Jolliffe D, Camargo CA, Sluyter J, et al. Vitamin D supplementation to prevent acute respiratory infections: systematic review and metaanalysis of aggregate data from randomised controlled trials. MedRx 2020.

39. Zhou YF, Luo BA, Qin LL. The association between vitamin D deficiency and community-acquired pneumonia: a meta-analysis of observational studies. Medicine (Baltimore) 2019;98(38):e17252. DOI: 10.1097/MD.0000000000017252.

40. Ilie PC, Stefanescu S, Smith L. The role of vitamin D in the prevention of coronavirus disease 2019 infection and mortality. Aging Clin Exp Res 2020;32(7):1195-1198. DOI: 10.1007/s40520-020-01570-8.

41. Mok CK, Ng YL, Ahidjo BA, et al. Calcitriol, the active form of vitamin D, is a promising candidate for COVID-19 prophylaxis. MedRxiv 2020. 